\title{
The tumor circadian clock: a new target for cancer therapy?
}

\author{
Silke Kiessling ${ }^{*}, 1$ \& Nicolas Cermakian ${ }^{2}$ \\ ${ }^{1}$ ZIEL - Institute of Food \& Health, Department of Nutrition \& Immunology, Technical University Munich, Freising, Germany \\ ${ }^{2}$ Douglas Mental Health University Institute, Department of Psychiatry, McGill University, Montreal, QC, Canada \\ * Author for correspondence: Tel.: +49 816171 2375; Fax: +49 816171 2824; silke.kiessling@tum.de
}

First draft submitted: 8 September 2017; Accepted for publication: 19 September 2017; Published online: 23 November 2017

Keywords: cancer therapy $\bullet$ circadian clock $\bullet$ tumor growth

Cancer is among the most frequent causes of death in humans. To date, established cancer therapies include surgery, radiation, chemo- and immunotherapy as well as hormonal treatments up to stem cell transplantation. In this editorial, we highlight an additional and so far underestimated factor involved in carcinogenesis and prognosis, namely biological clocks, which should be carefully considered to establish future cancer therapies. Disruption of the circadian clock was documented both in host-tissue and in cancer cells, and functional oscillations were associated with cancer prognosis and survival. We summarize recent advances in the field of chronobiology regarding the role of the host's and tumor-intrinsic circadian clock functions in carcinogenesis.

\section{The biological circadian clock controls cancer-related pathways}

A central clock in the hypothalamus and peripheral clocks in almost all organs and tissues altogether regulate physiological processes in a time of day dependent (circadian) manner. The molecular bases of circadian clocks are cellular oscillations generated by a number of rhythmically expressed interconnected clock genes [1]. Highthroughput experiments on murine tissues showed that in any given cell-type or organ, hundreds or thousands of genes are expressed with a circadian rhythm, and led to the estimation that around $50 \%$ of all genes oscillate in at least one organ [2]. As a consequence of this, most cellular and physiological processes show $24 \mathrm{~h}$ oscillations. This is the case of many aspects of the immune system, including many immune cells and responses of relevance to cancer [3]. Also, major regulators of the cell cycle and tumor suppressor genes were shown to be regulated by the circadian clock, such as WEE1, c-MYC and p21 [4]. Consistently, cell-cycle progression and proliferation show circadian regulation.

\section{Circadian clock disruption is associated with cancer}

Mismatch between the internal clock and the external time disrupts the circadian network, as reported for example in shift workers [5], and has been associated with a wide range of pathologies and disease states including cancer [4]. Mounting evidence from human-based epidemiological studies suggested an effect of circadian disruption during shift work on cancer risk. For example, the risk to develop breast cancer was extensively increased in nurses exposed to long-term rotating night shift work [6]. Similar results were obtained for prostate cancer risk in night shift workers [7]. Out of note, in 2007 an agency of the WHO has classified night shift work leading to circadian disruption as 'probably carcinogenic' to humans [8]. Since then, there has been growing interest in understanding how circadian disruption may play a role in cancer development and progression. In support of human studies, experimental work in rodents documented accelerated tumor growth when mice, engrafted with lung adenocarcinoma or Glasgow osteosarcoma, were exposed to repeated jet lag conditions [9,10]. Similarly, enhanced tumor development has been described in mice with genetic disruption of the circadian clock (although this is not the case for all clock gene mutants) [9,11-13] or surgical lesion of the central clock [14]. Taken together, circadian disruption coincides with increased carcinogenesis and faster tumor growth in a wide range of experimental setups. 


\section{Considering circadian rhythms in treatments}

Targeting the circadian clock may be a powerful tool to control tumorigenesis, since the literature reports that many cancer-related cellular processes, such as the cell cycle, cell proliferation, apoptosis and DNA damage are controlled by the circadian clock [15]. Interestingly, the targets of about half of the drugs currently on the market are encoded by genes showing circadian regulation [2]. In addition, the circadian regulation of processes such as metabolism or detoxification has severe consequences on the pharmacokinetics and pharmacodynamics of anticancer drugs, and thus, the outcome of anticancer therapies [16]. Therefore, chronotherapy, the principle of judiciously timed administration of therapeutic interventions, has been recognized as an important aspect in anticancer therapy [16]. The goal is to increase the treatment efficacy while minimizing toxicity [16]. For example, timed delivery of a therapeutic for colorectal cancer patients significantly improved the treatment efficacy and prolonged the patient's survival [17].

\section{Targeting the clock in tumors?}

Would it be possible to treat cancer by acting on circadian clocks? This is a question worth asking, given the circadian regulation of physiological systems relevant to cancer (e.g., immune responses, cell cycle, DNA repair), and the effects of circadian disruption on tumorigenesis (see the first two sections). Indeed, host circadian rhythms have often been found to be disrupted in cancer patients and mouse cancer models $[10,12,15,17]$. For example, in mice with lung adenocarcinoma, circadian gene expression was largely affected in the liver, with many genes losing rhythmicity, others becoming rhythmic, and some being shifted earlier or later [15]. In humans, alterations in clock gene expression and consequently clock-controlled gene expression was documented in the healthy gut tissues surrounding the tumor $[18,19]$.

However, the literature is much more abundant regarding circadian disruption within cancer cell lines and tumor tissues [13]. Notably, recent evidence indicates a significant reduction of circadian rhythmicity restricted to murine colon tumor tissue, whereas rhythms were unchanged in healthy colon tissue surrounding the tumor [19]. In fact, the intratumoral circadian disruption correlates with the patient's prognosis and survival $[17,18]$. Consequently, tumor cell-autonomous clock mechanisms may be contributing to the increased carcinogenesis rather than circadian rhythms in the host.

Is the tumor-intrinsic circadian clock involved in carcinogenesis? Daytime rather than nighttime administration of seliciclib, a cyclin-dependent kinase inhibitor, reduced Glasgow osteosarcoma progression, and concurrently improved the amplitude of clock gene expression rhythms within the tumor tissue [20]. Similarly, timed feeding, a major synchronizer of peripheral clocks, stimulated clock gene rhythmic expression in pancreatic adenocarcinoma, while reducing its growth speed [21]. Although these interesting animal studies were consistent with the idea that tumor-intrinsic circadian rhythms may impact on tumor growth, direct evidence was not provided.

In recent studies, two opposite approaches have been used to directly test a causative link between the tumor clock and its growth. Papagiannakopoulos and colleagues addressed the question by genetically 'switching off' the clock specifically in cancer cells [9]. Deleting Bmall or Per2 in a genetically-engineered mouse models of lung carcinoma led to increased proliferation in vitro and tumor formation in vivo. This is in keeping with the many reports showing faster proliferation in cultured cancer cells [13].

We have approached the question from a different angle. Instead of inactivating the tumor clock, we reasoned that since most cancer cell lines and various tumor tissues harbor a dysfunctional circadian clock, then rescuing the circadian clock in tumor cells might inhibit or slow down their growth [22]. We found suppressed clock gene expression in B16 mouse melanoma cells, and were able to restore rhythmic clock gene expression by various treatments such as dexamethasone (DEX), forskolin or heat shock. Each of these clock-inducing treatments significantly slowed down B16 cell proliferation. When used to generate subcutaneous solid tumors in mice, the B16 cells did not show clock gene rhythms in vivo unless they were injected with DEX. This treatment both restored clock function and dramatically slowed down tumor growth (about two-third reduction after only 1 week) [22]. This was not due to an effect on the host-immune response, and moreover, inhibition of the tumor clock by shRNA-mediated knockdown completely prevented the effects of DEX, implying that they were happening via the tumor clock. Similar results were found in another cancer cell-type, the human colon carcinoma HCT-116 cells, pointing to the broader applicability of the concept [22].

What is the molecular mechanism underlying the clock's control over tumor growth? In contrast to healthy cells with a cell-division rate of about $24 \mathrm{~h}$, cancer cells are characterized by uncontrolled fast cell division. In fact, the literature reports that cell-cycle gene expression is altered in cancer cells $[11,15]$ and that increased levels of the 
proto-oncogene Myc, upon mutation of clock genes in tumor cells, correlate with the cell-division speed [9]. Since the cell cycle is under circadian control, cancer cells might enhance their proliferation through manipulation of the circadian control over the cell cycle. Notably, our evidence indicates that the reduced proliferation and tumor growth due to activation of the tumor clock is achieved by inducing oscillations of cell-cycle genes important for the G1-to-S transition, such as $c-M y c$, Cyclin $E$ and $p 21$, which subsequently decreases the entry in $S$ phase [22]. Taken together, current literature supports the strategy of acting on the tumor clock in order to control tumor progression.

\section{Conclusion \& future perspective}

Slowing down tumor growth by manipulating the tumor circadian clock may critically grant more time for established anticancer approaches, such as chemotherapy and surgical tumor resection. Thus, this new idea of acting on the tumor clock might represent an innovative strategy to improve the outcome of the treatments and potentially the patient's survival. Additionally, the development of long-term strategies to strengthen circadian rhythms in populations at risk, such as shift workers, may prevent the incidence of cancer.

Financial \& competing interests disclosure

This work was funded by the Canadian Institutes for Health Research (grant MOP-119322, to N Cermakian). The authors have no relevant affiliations or financial involvement with any organization or entity with a financial interest in or financial conflict with the subject matter or materials discussed in the manuscript. This includes employment, consultancies, honoraria, stock ownership or options, expert testimony, grants or patents received or pending, or royalties.

No writing assistance was utilized in the production of this manuscript.

\section{References}

Papers of special note have been highlighted as: $\bullet$ of interest; $\bullet \bullet$ of considerable interest

1. Partch CL, Green CB, Takahashi JS. Molecular architecture of the mammalian circadian clock. Trends Cell Biol. 24(2), 90-99 (2014).

2. Zhang R, Lahens NF, Ballance HI, Hughes ME, Hogenesch JB. A circadian gene expression atlas in mammals: implications for biology and medicine. Proc. Natl Acad. Sci. USA 111(45), 16219-16224 (2014).

3. Labrecque N, Cermakian N. Circadian clocks in the immune system. J. Biol. Rhythms 30(4), 277-290 (2015).

4. Feillet C, Van Der Horst GT, Levi F, Rand DA, Delaunay F. Coupling between the circadian clock and cell cycle oscillators: implication for healthy cells and malignant growth. Front. Neurol. 6, 96 (2015).

- An up-to date review about the link between the circadian clock and the cell cycle.

5. Kiessling S, Eichele G, Oster H. Adrenal glucocorticoids have a key role in circadian resynchronization in a mouse model of jet lag. J. Clin. Invest. 120(7), 2600-2609 (2010).

6. Wegrzyn LR, Tamimi RM, Rosner BA et al. Rotating night shift work and risk of breast cancer in the Nurses' Health Studies. Am. J. Epidemiol. 186(5), 532-540 (2017).

7. Papantoniou K, Castano-Vinyals G, Espinosa A et al. Night shift work, chronotype and prostate cancer risk in the MCC-Spain case-control study. Int. J. Cancer 137(5), 1147-1157 (2015).

- The most recent report about the prostate cancer risk in shift workers.

8. Straif K, Baan R, Grosse Y et al. Carcinogenicity of shift-work, painting, and fire-fighting. Lancet Oncol. 8(12), 1065-1066 (2007).

- The first official statement from the World Health Organization that shiftwork can be carcinogenic.

9. Papagiannakopoulos T, Bauer MR, Davidson SM et al. Circadian rhythm disruption promotes lung tumorigenesis. Cell Metab. 24(2), 324-331 (2016).

-. One of the few studies directly addressing the causal effects between tumor circadian clock disruption and tumorigenesis, both in vitro and in tumors in mice.

10. Filipski E, Delaunay F, King VM et al. Effects of chronic jet lag on tumor progression in mice. Cancer Res. 64(21), 7879-7885 (2004).

11. Zeng ZL, Wu MW, Sun J et al. Effects of the biological clock gene Bmall on tumour growth and anti-cancer drug activity. J. Biochem. 148(3), 319-326 (2010).

12. Mteyrek A, Filipski E, Guettier C, Okyar A, Levi F. Clock gene Per2 as a controller of liver carcinogenesis. Oncotarget 7(52), 85832-85847 (2016).

13. Kiessling S, Cermakian N. Clock genes and cancer. In: Biological Timekeeping: Clocks, Rhythms and Behaviour. Kumar V (Ed.). Springer India, New Delhi, India, 481-500 (2017).

14. Filipski E, King VM, Li X et al. Host circadian clock as a control point in tumor progression. J. Natl Cancer Inst. 94(9), 690-697 (2002). 
15. Masri S, Cervantes M, Sassone-Corsi P. The circadian clock and cell cycle: interconnected biological circuits. Curr. Opin. Cell Biol. 25(6), 730-734 (2013).

16. Ballesta A, Innominato PF, Dallmann R, Rand DA, Levi FA. Systems chronotherapeutics. Pharmacolog. Rev. 69(2), 161-199 (2017).

17. Innominato PF, Levi FA, Bjarnason GA. Chronotherapy and the molecular clock: clinical implications in oncology. Adv. Drug Delivery Rev. 62(9-10), 979-1001 (2010)

18. Oshima T, Takenoshita S, Akaike $\mathrm{M}$ et al. Expression of circadian genes correlates with liver metastasis and outcomes in colorectal cancer. Oncol. Rep. 25(5), 1439-1446 (2011).

19. Sotak M, Polidarova L, Ergang P, Sumova A, Pacha J. An association between clock genes and clock-controlled cell cycle genes in murine colorectal tumors. Int. J. Cancer 132(5), 1032-1041 (2013).

20. Iurisci I, Filipski E, Reinhardt J et al. Improved tumor control through circadian clock induction by Seliciclib, a cyclin-dependent kinase inhibitor. Cancer Res. 66(22), 10720-10728 (2006).

-. A key study correlating tumor growth with clock gene rhythms activation in tumors following the timed administration of an anti-cancer drug in mice.

21. Li XM, Delaunay F, Dulong S et al. Cancer inhibition through circadian reprogramming of tumor transcriptome with meal timing. Cancer Res. 70(8), 3351-3360 (2010).

22. Kiessling S, Beaulieu-Laroche L, Blum ID et al. Enhancing circadian clock function in cancer cells inhibits tumor growth. BMC Biol. 15(1), 13 (2017).

-. A key study testing the causal relationship between the tumor clock and the growth speed of the tumor. Specifically, it shows that activating circadian clock gene expression in tumor cells slows down proliferation and tumor growth. 